\title{
Dielectric-metal triboelectric nanogenerators for ocean wave impact self-powered applications
}

\author{
Ulises Tronco Jurado, Suan Hui Pu, Member, IEEE, and Neil M. White, Senior Member, IEEE
}

\begin{abstract}
This paper describes the effect of oscillatory frequencies caused by ocean wave impact on the output performance of dielectric-metal contact separation mode triboelectric nanogenerators (DMCS-TENG). The triboelectric effect is generated as a result of regular, non-uniform contact between a dielectric layer which gains electrons (negative triboelectric material) and a conductive layer that loses electrons (positive triboelectric material). Impact testing was used to characterize arc-shaped dielectric-metal single electrode triboelectric nanogenerators (DMSE-TENG) with different triboelectric material combinations based in their output power generation, using a $40 \mathrm{~mm}$ ball bearing to apply a $12 \mathrm{~N}$ force impulse. It was found that the best dielectric-conductor combination for the DMCS-TENG performance was polyimide and PDMS in contact with the conductor layers of aluminium and silver conductive cloth tape. Therefore, in the range of operation from $30 \mathrm{~Hz}$ to $300 \mathrm{~Hz}$ with an amplitude acceleration of 319.62 to $559.29 \mathrm{~mm} / \mathrm{s}^{2}$. The maximum generated output power, power density and total energy conversion efficiency of the device, made with polyimide and honeycomb patterned aluminium foil, can reach up to $778.43 \mu \mathrm{W}, 12.16 \mu \mathrm{W} / \mathrm{cm}^{2}$ and $15.85 \%$ respectively for a load resistance of $10 \mathrm{M} \Omega$. The output power performance of the DMCS-TENG shows an enhancement by a factor of 2.3 with a honeycomb-patterned aluminium foil, by increasing the surface charge density between the layers in contact, relative to flat aluminium foil. Additionally, through the integration of the energy harvester prototype into a water wave generator tank. An output power density of $169.218 \mathrm{~mW} / \mathrm{m}^{2}$ was reached, where it is expected to generate output power energy around $3.05 \mathrm{~W}$, over an area of $18 \mathrm{~m}^{2}$ with wave sizes among $0.3 \mathrm{~m}$ to $4 \mathrm{~m}$. This work demonstrates that the device can function as an energy harvesting mechanism for ocean wave sensing applications that require selfpowering.
\end{abstract}

Index Terms - ocean wave impact; compressed air bubbles; oscillatory frequencies; triboelectric effect; dielectric-conductor; ocean wave sensing; self-powering.

\section{INTRODUCTION}

$\mathrm{O}$ CEAN wave energy has been attracting a great interest as a source of electrical energy for many years [1]. Effective utilization and sustainability of this kind of energy can be exploited due to the effects of climate change with the rapid increase of sea levels and recurrent flooding of coastal zones. Specifically, the cumulative loads of wave impact forces at coastal defence structures deliver high magnitude pulse-like

A conference paper related with this research project was presented at the IEEE Sensors 2017 Conference, Glasgow, UK, and was published in its Proceedings.

Ulises Tronco Jurado is with Mechatronics Research Group and Smart Electronic Materials and Systems Research Group, University of Southampton, SO17 1BJ, UK (e-mail: utj1n15@soton.ac.uk).

Suan Hui $\mathrm{Pu}$ is with Mechatronics Research Group, University of Southampton, SO17 1BJ, UK, and University of Southampton Malaysia forces with a wide frequency range of oscillatory compressed air bubbles at structure-water interfaces. The surging flow front impacts the structure, spreads vertically upwards as shown in Fig. 1 (a), and produces the first hydrostatic pressure oscillations peak between 30 to $80 \mathrm{~Hz}$ with an amplitude range of 57 to $116 \mathrm{~Pa}$. After the wave collapses a plunging breaker is formed (Fig. 1 (b) and many air bubbles are entrained (Fig. 1 (c)), which compresses and dilates in a high frequency oscillatory manner, impinging upon the coastal protective structure as shown in Fig. 1 (d). This yields a cyclic hydrostatic pressure response between 150 to $252 \mathrm{~Hz}$ with an amplitude range of 148 to $348 \mathrm{~Pa}$, predicted through a fluidstructure simulation $[2,3]$. Such energy can be potentially harvested and exploited using a dielectric-metal contact separation mode triboelectric nanogenerators (DMCS-TENG) as a new type of energy harvester for ocean wave impacts (Fig. 1 (a)). This aim in the main frequencies $30,80,150,180,200$, 219 and $252 \mathrm{~Hz}$ to harvest the major energy $[2,3]$. The triboelectric effect is generated by regular non-uniform contact between a dielectric layer that gains electrons (negative

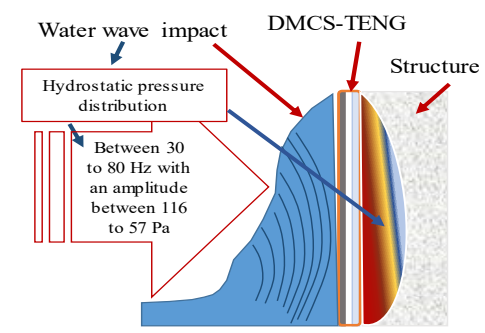

(a)

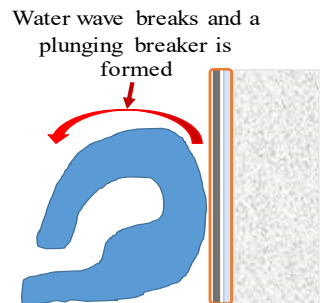

(b)

Air bubbles compresses and dilates impinging upon the DMCS-TENG and the coastal $\downarrow$ protective structure

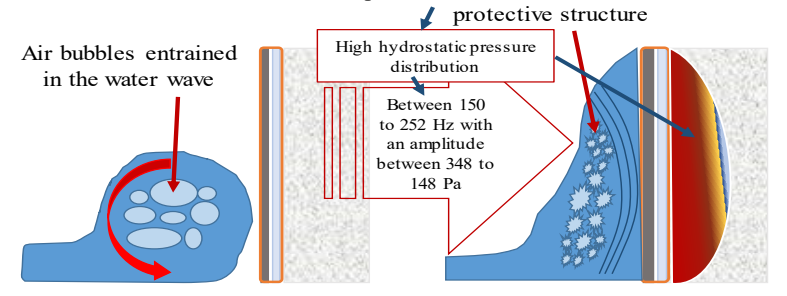

(c) (d)
Fig. 1. (a-d) Hydrostatic pressure distribution of the ocean wave impact at the water-structure interface and main frequencies of interest $(30,80,150,200,219$ and $252 \mathrm{~Hz}$ ) [2, 3] for ocean wave energy harvesting generated by (a) the water wave impact and (d) the impact of oscillatory compressed air bubbles flows at the water-structure interface exploited using the DMCS-TENG.

Campus, Iskander Puteri 79200, Johor, Malaysia (e-mail: suanhui.pu@soton.ac.uk).

Neil M. White is with Smart Electronic Materials and Systems Research Group, University of Southampton, SO17 1BJ, UK (e-mail: nmw@ecs.soton.ac.uk). 
triboelectric material) and a conductive layer that loses electrons (positive triboelectric material), where the output performance changes in proportion to the mechanical energy applied by pressure oscillation [4]. TENG can be used as energy harvesters for self-powering sensors $[5,6]$. Their advantages include broadband behaviour, lightweight, high energy density and ease of fabrication exploiting low-cost, readily available materials [7]. Firstly, in order to study and to find the most promising triboelectric material combinations based on their output power generation to enhance the DMCS-TENG performance for mechanical energy harvesting, arc-shaped dielectric-metal single electrode triboelectric nanogenerators (DMSE-TENG) were fabricated. The output performance characterization using contact electrification between different dielectric-metal combinations was performed through an impact test. Secondly, in this study we have developed a finite element model (FEM) for a DMCS-TENG using COMSOL Multiphysics, to understand its electromechanical characteristics. Thirdly, the output performance of the fabricated DMCS-TENG using the best triboelectric material combination was evaluated at the frequencies mentioned above. Finally, the proposed energy harvester that shows the highest output performance was tested in a water wave generator tank, to analyse its performance in realistic conditions. It is expected that the enhanced output performance of the proposed energy harvesting prototypes will enable future advancement in energy harvesting at structure-water interfaces for ocean wave monitoring applications that require self-powering sensors.

\section{Triboelectric Material Selection Using DMSe-Teng}

\section{A. DMSE-TENG fabrication and electrical characterization} set-up

In order to maximize the DMCS-TENG performance for mechanical energy harvesting from the oscillatory frequencies generated by wave impact, arc-shaped dielectric-metal single electrode triboelectric nanogenerators (DMSE-TENG) were fabricated using different material combinations. The arc shaped DMSE-TENG, as illustrated in Fig. 2 (prototype 1), were fabricated using a simple, low-cost process. The device prototypes have an active triboelectric area of $5 \mathrm{~cm} \mathrm{x} 5 \mathrm{~cm}$, and an acrylic sheet was selected as a substrate material. The conductor layer (either aluminium, copper or silver) was attached to the acrylic with a thin adhesive layer, at room temperature. The conductor layer is used as a triboelectric material, and an electrode connected to an external load of 10 $\mathrm{M} \Omega$ for electrical characterization. Likewise, the dielectric layer (polyimide, PDMS, polytetrafluoroethylene, i.e. PTFE, Teflon FEP etc.) was fixed with thin adhesive on a clear acetate film (thickness $=100 \mu \mathrm{m}$ ). The dielectric layer was attached with an arc-shaped form to the metal electrode using Kapton tape $(1 \mathrm{~cm}$ width) at room temperature. The maximum gap

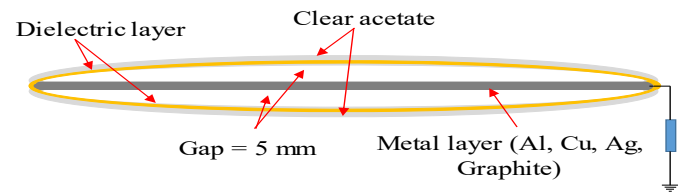

Fig. 2. Two-dimensional schematic of the arc-shaped DMSE-TENG (Prototype $1)$.

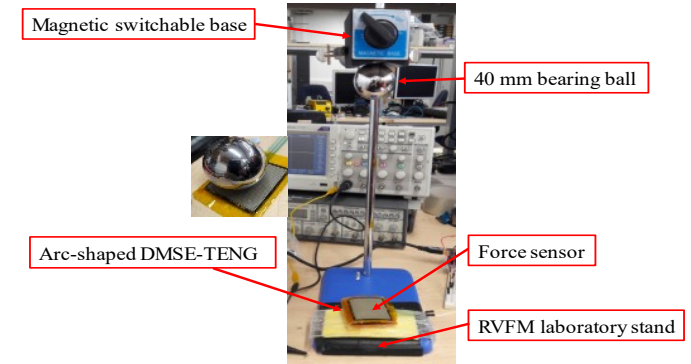

Fig. 3. Experimental set-up of impact test using a $40 \mathrm{~mm}$ ball bearing on the arc-shaped DMSE-TENG prototypes (connected to a $10 \mathrm{M} \Omega$ load resistor) with different triboelectric materials combinations.

between the arc-shaped dielectric layer and the metal layer was $5 \mathrm{~mm}$. The materials used for the arc-shaped DMSE-TENG prototypes fabrication are shown in Table I (Literature).

The output power performance characterization of the arcshaped DMSE-TENG prototypes was evaluated from the generated output voltage and current, measured with an impact test set-up in order to study and find the optimum triboelectric material combination to reach the highest output performance. This system uses a $40 \mathrm{~mm}$ ball bearing (mass $=261.04$ grams) attached to a magnetic switchable base, which is fastened to a RVFM laboratory stand set at the height of $30 \mathrm{~cm}$. The device prototype was held at the base of the laboratory stand and the applied force to it was measured using a force sensor (Interlink electronics FSR406 1.5" diameter force sensing resistor) as shown in Fig. 3. The output voltage measurements of the arcshaped DMSE-TENG were obtained with a digital oscilloscope Tektronix TDS 2014C, the output current measurements were performed using an Agilent Technologies N6705B power analyser.

\section{B. Electrical characterization of the arc-shaped DMSE-TENG for the triboelectric material optimization}

The output performance measurements of the arc-shaped DMSE-TENG prototypes through the impact test, which applies a force impulse of $12 \mathrm{~N}$. The results show that the maximum output power was $6.28 \mu \mathrm{W}$ corresponding to an output voltage $\left(V_{R M S}\right)$ of $5.22 \mathrm{~V}$ and current $\left(I_{R M S}\right)$ of $1.20 \mu \mathrm{A}$ using a triboelectric material combination of PDMS-silver conductive cloth tape. The instantaneous voltage of the sample using PDMS in contact with aluminum under the impact of a 40 $\mathrm{mm}$ ball bearing is shown in Fig. 4 .

The highest instantaneous output voltage occurs when the dielectric layer is in contact with the conductor layer. The movement of the top dielectric layer due to the impact of the ball bearing, changes the local electrical field distribution created between both layers due the surface charge transfer, so that there is electron exchange between the bottom electrode, external load of $10 \mathrm{M} \Omega$ and the ground to maintain the potential

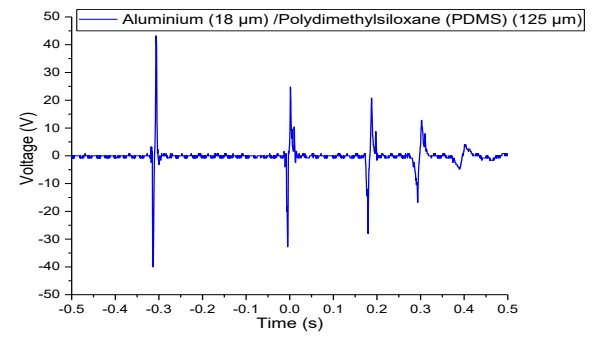

Fig. 4. Instantaneous output voltage of arc-shaped DMSE-TENG prototype (connected to a $10 \mathrm{M} \Omega$ load resistor) using PDMS in contact with Aluminum under the impact of a $40 \mathrm{~mm}$ ball bearing. 
change of the electrode. The first impact of the ball bearing generates the highest voltage peak, which is related with the impact force that helps to increase the contact area between both layers. The instantaneous voltage decreases due the bouncing behavior of the ball bearing after impact with the sample as shown in Fig. 4. Among all the arc-shaped DMSE-TENG prototypes the top five highest output power performance was dominated by structures with silver conductive cloth tape in contact with polyimide, PDMS, silicone rubber and PET except PTFE, where the highest performance was in contact with aluminium. The dynamic operation of the DMSE-TENG prototype is shown in the Supplementary Information document.

The output performance comparison of arc-shaped DMSETENG devices fabricated with silver conductive cloth tape versus the ones fabricated using aluminum foil is shown in Fig. 5 . The effective values of output voltage ( $V_{\mathrm{RMS}}$ of $1.22 \mathrm{~V}$ to 5.96 $\mathrm{V})$, current $\left(I_{\mathrm{RMS}}\right.$ of $0.49 \mu \mathrm{A}$ to $\left.1.20 \mu \mathrm{A}\right)$, output power $(0.668$ $\mu \mathrm{W}$ to $6.284 \mu \mathrm{W})$ and its standard deviation analysis calculated for each prototype (10 measurements performed on each sample). Such values showed that the highest performance of the proposed triboelectric energy harvesting devices can be achieved by using silver conductive cloth tape in contact with the dielectric layers of PDMS and polyimide. Furthermore, the polyimide or PTFE-silver conductive cloth tape or aluminium foil combinations can reach similar output power performance, ranging from $3.8 \mu \mathrm{W}$ to $5.8 \mu \mathrm{W}$.

The ranking of the triboelectric materials used for the arcshaped DMSE-TENG prototypes, arranged according to the output performance is shown in Table I (Tested). The most positive material of the conductors according the measurements, was shown to be the silver conductive cloth tape and the most negative material was PDMS. This means that the charge transferred using this material combination will be the highest from the selection of triboelectric materials. Each conductor material on Table I (Tested) was highlighted with a specific color in order to identify the higher output performance, when they are in contact with the insulators highlighted with the same color. The dominance of the silver tape among the chosen triboelectric materials, with the aim of reaching higher electrical output performance in the proposed energy harvesting prototypes, is clearly seen. Although silver conductive cloth is the best positive material, Al foil was chosen

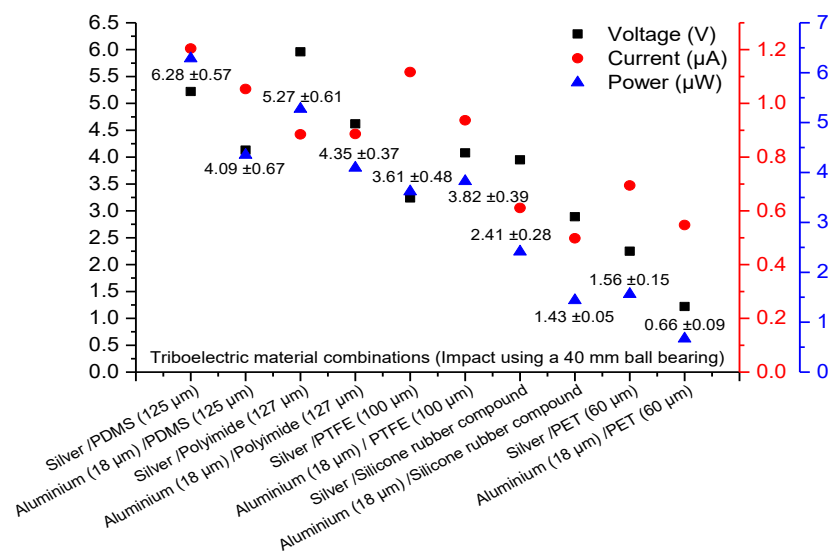

Fig. 5. Comparison of the output voltage $\left(V_{R M S}\right)$, current $\left(I_{R M S}\right)$ and power effective values of the top five arc-shaped DMSE-TENG fabricated prototypes (connected to a $10 \mathrm{M} \Omega$ load resistor) with silver conductive cloth tape and aluminum foil, under impact test through a $40 \mathrm{~mm}$ ball bearing.
TABLE I

COMPARISON OF THE RANKINGS OF POSITIVE AND NEGATIVE TRIBOELECTRIC MATERIALS IN TERMS OF EASE OF LOSING OR GAINING ELECTRONS WITH DATA FROM THE LITERATURE [14] VERSUS OUR IMPACT TEST CHARACTERIZATION DATA.

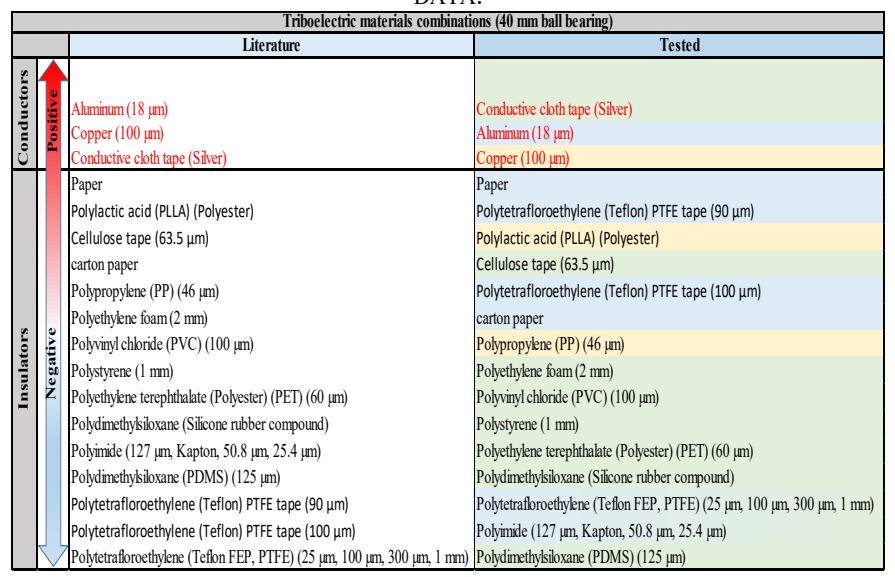

for the fabrication of the DMCS-TENG as a low-cost option with excellent performance.

\section{DMCS-TENG FEM MODEL}

Considering the so called "V-Q-X relationship" proposed for the operation and modelling of a TENG when it is connected with a resistive load (R), utilizing Kirchhoff's law, the governing equation of the whole system can be given by [8]:

$$
R \frac{d Q}{d t}=-\frac{1}{C(x)} Q+\operatorname{Voc}(x)=-\frac{1}{C} Q+V_{O C}
$$

Where $V_{O C}$ is the voltage generated between two DMCSTENG contacts, $Q$ is the amount of charge between the two contacts, $C$ is the capacitance between the two electrodes, and $x$ is the distance between the DMCS-TENG triboelectric layers that varies with the mechanical energy and phase of motion at time, $t$. According to these models, the power output performance by the DMCS-TENG depends on the output current $(I)$. The voltage $\left(V_{O C}\right)$ is influenced by the load resistance, and the surface charge density $\sigma$ depends on the properties of the dielectric materials. The maximum theoretical surface charge density $\sigma_{\max }$ for contact DMCS-TENG was determined using the ion injection method, by comparing the threshold voltage for the air breakdown $\left(V_{a-b}\right)$ and the actual voltage drop $\left(V_{\text {gap }}\right)$ across the air gap. Thus, when $x$ starts to increase from 0 during the releasing half cycle of the DMCSTENG, the $V_{a-b}$ at any $x>0$ needs to remain bigger than $V_{\text {gap }}$ in order to avoid breakdown of air.

$$
V_{a-b}-V_{g a p}>0=\frac{A(P x)}{\ln (P x)+B}-\frac{d \sigma x}{\varepsilon_{0}\left(d+x \varepsilon_{r}\right)}>0
$$

where $P$ is the gas pressure for air at standard atmospheric pressure of $101 \mathrm{kPa}, A$ and $B$ are the constants determined by the pressure of the gas, $A=2.87 \mathrm{X} 10^{5} \mathrm{~V} /(\mathrm{atm} \cdot \mathrm{m})$, and $B=12.6$. $\varepsilon_{r}$ is the relative permittivity of the dielectric layer, and $\varepsilon_{0}$ is the vacuum permittivity. From equation $(2), \sigma_{\max }$ can be determined as follows [9]:

$$
\sigma_{\max }=\left[\frac{A P \varepsilon_{0}\left(d+x \varepsilon_{r}\right)}{d(\ln (P x+B)}\right] \min
$$




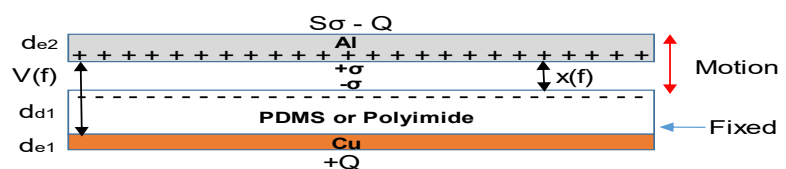

Fig. 6. Two-dimensional schematic of the DMCS-TENG prototype (Prototype 2).

The FEM simulations were performed coupling electromechanics and electrical circuit interfaces, to model the current, voltage and output power generated by the DMCSTENG connected to resistive loads (varying from $1 \mathrm{M} \Omega$ to 100 $\mathrm{M} \Omega$ ) under the oscillating frequencies estimated of the ocean wave impact forces (Fig. 1). These are based on the DMCSTENG structure (Prototype 2), as shown in Fig. 6. Two structures comprising different dielectric layers, namely polydimethylsiloxane (PDMS, thickness $d_{d l}=125 \mu \mathrm{m}, \varepsilon_{r}=2.5$ ) and polyimide (Kapton, thickness $d_{d l}=50.8 \mu \mathrm{m}, \varepsilon_{r}=3.4$ ), were attached to a copper $\left(\mathrm{Cu}\right.$, thickness $\left.d_{e l}=100 \mu \mathrm{m}\right)$ lower electrode 1 . An aluminum (Al, thickness $\left.d_{e 2}=100 \mu \mathrm{m}\right)$ layer was used as the second triboelectric material, the top electrode 2 (grounded). The top layer was fixed in both extreme sides and is the point at which the periodic motion ( six cycles $(\mathrm{t}=0.12$ s)) was applied with the oscillatory frequencies of $30,80,150$, 200, 219 and $250 \mathrm{~Hz}$ and an amplitude of $1 \mathrm{~mm}$.

From equation (3), we consider a scenario where the maximum gap $x$ separation is $1 \mathrm{~mm}$ and the time duration is 30 seconds. The two tribo-charge surfaces were assigned to the model with a calculated value $\sigma_{P D M S}=\sigma_{\max , P D M S}= \pm 11.53 \mu \mathrm{C} / \mathrm{m}^{2}$ and $\sigma_{\text {max,Polyimide }}= \pm 34.06 \mu \mathrm{C} / \mathrm{m}^{2}$ (see Supplementary Information document). A series of simulations were performed in order to analyze the output performance of the entire system,
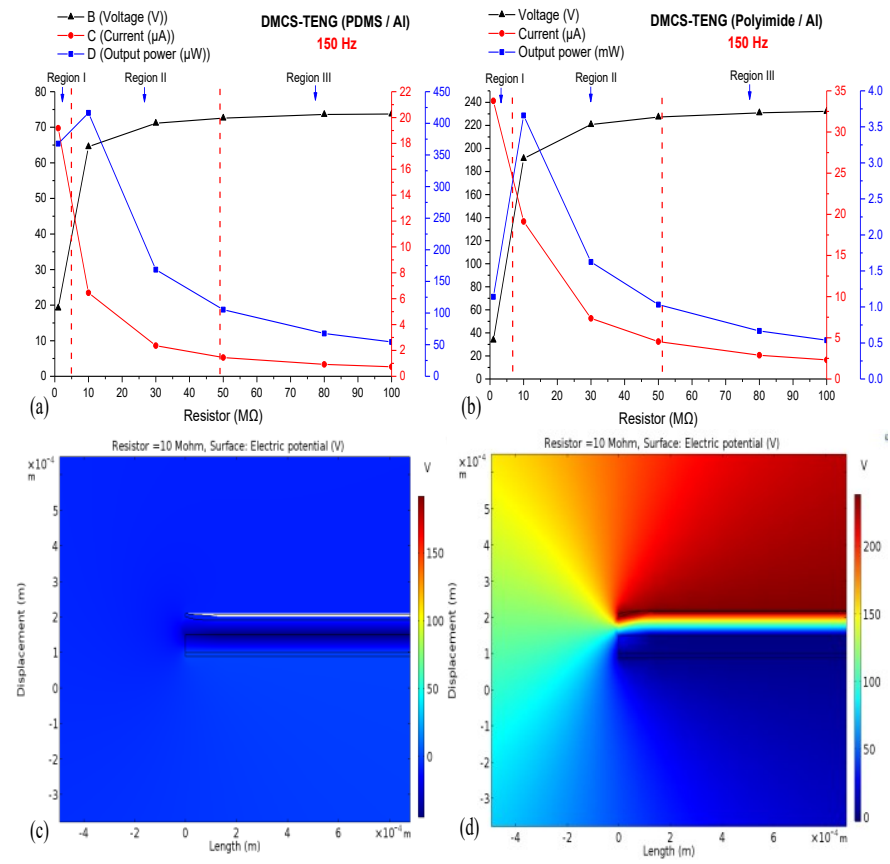

(c)

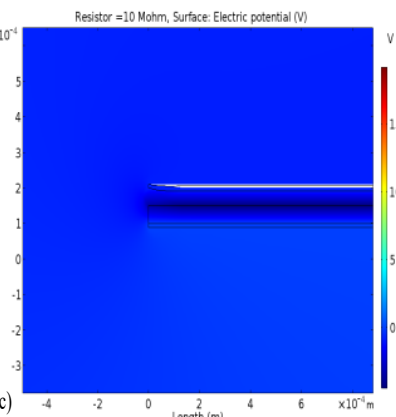

Fig. 7. The influence of the load resistance divided into three working regions on the magnitude of the output voltage $(V)$, current $(I)$ and maximum output power performance of the DMCS-TENG prototype $(L=8 \mathrm{~cm})$ with (a) PDMS$\mathrm{Al}$ and (b) Polyimide-Al layers at the oscillation frequency of $150 \mathrm{~Hz}$. DMCSTENG under oscillatory frequency and deformation. (c) Two dimensional shape showing the two triboelectric layers in contact, (d) Al layer released, which will rise a much higher potential.

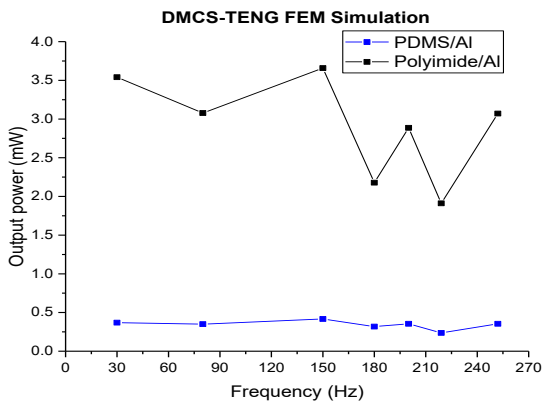

Fig. 8. Maximum instantaneous output power of simulated DMCS-TENG prototypes as a function of the main oscillatory frequencies of $30,80,150,180$, 200, 219 and $252 \mathrm{~Hz}$ with an amplitude of $1 \mathrm{~mm}(\mathrm{t}=0.12 \mathrm{~s})$.

with different structure lengths $(L=2.5 \mathrm{~cm}, 5 \mathrm{~cm}$ and $8 \mathrm{~cm})$ over the oscillatory frequency range mentioned earlier, so that the best design can be found. The highest calculated output performance for both samples was obtained with the DMCSTENG structure of $L=8 \mathrm{~cm}$ at the oscillation frequency of 150 $\mathrm{Hz}$ as shown in the three regions of operation [8] of the proposed devices in Fig. 7 (a) and Fig. 7 (b). The DMCS-TENG with polyimide-Al reached a higher maximum instantaneous output power $=3.66 \mathrm{~mW}$ with a $10 \mathrm{M} \Omega$ load shown in Fig. 7 (b). In comparison, the maximum instantaneous output power of the DMCS-TENG with PDMS-Al was $416.47 \mu \mathrm{W}$ in region II as depicted in Fig. 7 (a).

DMCS-TENG in the original state before the contact of the triboelectric layers do not experience charge transfer, and thus no electrical potential occurs across the electrodes (Fig. 7 (c)). By applying a force to the upper metal layer on the dielectric, each layer will be brought into surface contact, resulting in electron transfer from a material in the positive side of the triboelectric series to the one in the negative side in the series [10]. Accordingly, electrons will be injected from the Al to the polyimide/PDMS surface, leaving the positive charges on the Al layer. Such a process will continue in the first hundreds of cycles until the accumulated charges reach a saturation and equilibrium, and the negative charges will be preserved on the dielectric surface due to the nature of the insulator [11].

Once the pressing force is released, the Al layer of the DMCSTENG will quickly rebound back due to the elasticity of the film and a gap is formed again between the two layers. From the numerical simulation results, it can be seen that the electric field generated by separate surface charges will give rise to a much higher potential on the Al layer side than the bottom electrode (Fig. 7 (d)). Such potential difference will drive the flow of positive charges from Al layer to the bottom electrode through the external load until the potential difference is fully offset by the transferred charges, rendering the bottom electrode with a surface charge density of $+Q$, while the charges on the $\mathrm{Al}$ is with a sum of two parts. One part is due to the transferred charges between electrode 1 and electrode $2(-Q)$ and the other part is due to the triboelectric charges at the surface area $(S \sigma)$. Thus, the total charges on electrode 2 are $S \sigma-Q$. Subsequently, when the two layers in the DMCS-TENG are in contact again, charge redistribution will result in a positive potential on the bottom electrode, which will drive all of the transferred charges $+Q$ to flow back to the Al layer. The complete electricity generation cycle is achieved and the device will go back to the equilibrium state. 
The maximum output power of both energy harvesters is at $150 \mathrm{~Hz}$, followed by $30 \mathrm{~Hz}$ under the load resistor of $10 \mathrm{M} \Omega$ (between $3.7 \mathrm{~mW}$ to $230 \mu \mathrm{W}$ ), which tends to decrease and fluctuate as the operation cycle increases. The difference in the output power of the two simulated DMCS-TENG prototypes across the oscillation frequency range $(30$ to $252 \mathrm{~Hz}$ ) is shown in Fig. 8. Such difference is related to the higher surface charge density at the device with polyimide-Al due the thinner dielectric layer.

\section{DMCS-TENG ELECTRICAL CHARACTERIZATION UNDER DIFFERENT OSCILLATING FREQUENCIES AND USING A WATER WAVE GENERATOR TANK}

\section{A. DMCS-TENG fabrication and electrical characterization set-up}

For preliminary triboelectric material characterization using the impact tests, five DMCS-TENG prototypes of similar configuration to those used in the COMSOL model (Fig. 6) were fabricated using the most promising triboelectric material combinations that showed the highest output power. The DMCS- TENG prototypes have an active triboelectric area of 8 $\mathrm{cm} \times 8 \mathrm{~cm}$. Two types of aluminium foil were evaluated, one flat aluminium foil (with $t_{e 2}=18 \mu \mathrm{m}$ ) and a honeycomb patterned aluminium foil (with $t_{e 2}=16 \mu \mathrm{m}$, individual hexagon area $=31.83 \mathrm{~mm}^{2}$ ). The aluminium foil were fixed with a thin adhesive layer on the acrylic substrates at room temperature, acting as both the triboelectric material and the electrode 2 that is grounded. The dielectric layers (PDMS $\left(d_{\mathrm{d} 1}=125 \mu \mathrm{m}\right)$, silicone rubber $\left(d_{\mathrm{d} 1}=150 \mu \mathrm{m}\right), \operatorname{FEP}\left(d_{\mathrm{d} 1}=25 \mu \mathrm{m}\right)$, polyimide $\left(d_{\mathrm{d} 1}=127 \mu \mathrm{m}\right)$ and $\left.\operatorname{PET}\left(d_{\mathrm{d} 1}=60 \mu \mathrm{m}\right)\right)$ were fixed with conductive acrylic adhesive (thickness $=25 \mu \mathrm{m}$ ) at room temperature on a copper layer $\left(t_{e l}=100 \mu \mathrm{m}\right)$ connected to an external load of $10 \mathrm{M} \Omega$ for electrical characterization. The honeycomb patterned aluminium foil was selected to improve the contact electrification due to its surface morphology. Surface patterning is potentially a low-cost option to enhance the output performance of the DMCS-TENG prototypes [1214].

The output performance response of the fabricated DMCSTENG devices (focused in the frequencies of interest 30, 80, 150, 200, 219 and $252 \mathrm{~Hz}$ ) was characterized using a Pasco Scientific SF-9324 mechanical shaker table connected to a TG550 function generator in order to replicate the conditions of the mechanical energy generated by the oscillating frequencies caused by ocean wave impacts. The function generator output was configured as a sine wave and the frequency swept from 25 to $300 \mathrm{~Hz}$ with an increasing step of $5 \mathrm{~Hz}$ for 55 seconds. The driving amplitude was set at $20 \mathrm{~V}$ peak-to-peak. The $\mathrm{Al}$ electrode was placed facing the surface of the dielectric layer and bound together on each side at the middle with Kapton tape (1 cm width) at room temperature (Fig 9 (a)). Finally, each of the DMCS-TENG prototypes were mounted as depicted in Fig 9 (b). The gap of the DMCS-TENG varies with the mechanical oscillation producing the triboelectric effect when the dielectric layer is in non-uniform contact with the Al film. The average acceleration, velocity and displacement generated by the mechanical shaker on the proposed energy harvester prototypes were measured with a PDV-100 Portable Digital Vibrometer.

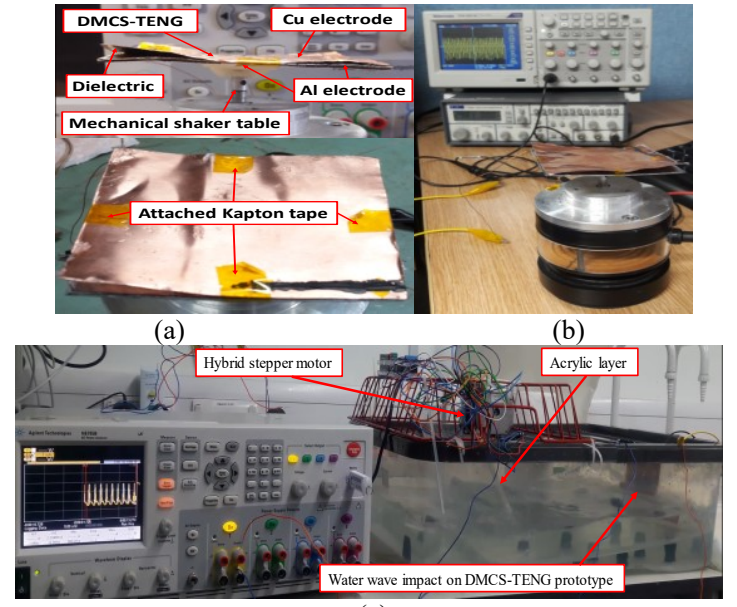

(c)

Fig. 9. (a) Fabricated DMCS-TENG, (b) DMCS-TENG mounted on the mechanical shaker table for the output performance measurements at different frequencies of oscillation. (c) Insulated DMCS-TENG prototype placed on the water wave generator tank for its output performance measurements.

The energy harvester prototype which shows the higher output power performance through the aforementioned experiment, was characterized in water conditions using a wave generator tank, in order to simulate the real conditions of the mechanical energy generated by ocean wave impacts. A hybrid stepper motor (RS Pro 535-0502) attached with an acrylic layer of $20 \mathrm{~cm} \times 20 \mathrm{~cm}(\mathrm{t}=8 \mathrm{~mm})$ is used to generate the water waves motion into the water wave tank mounted on the left side of the tank [15]. The DMCS-TENG prototype was insulated with a Polyethylene plastic pack, in order to protect it from the contact with water. Such device was placed at the wall on the right side of the tank facing the impact of the water wave as shown in Fig. 9(c) where the water wave breaks with an amplitude of $10 \mathrm{~cm}$ and frequency of $1.20 \mathrm{~Hz}$. The output power generated by the DMCS-TENG prototypes was calculated through the output voltage, and output current measurements performed using a digital oscilloscope Tektronix TDS 2014C and an Agilent Technologies N6705B Power analyser.

\section{B. DMCS-TENG dynamic operation, experimental results and discussion}

The analytical model to calculate the maximum open-circuit voltage at the resonant frequency of the DMCS-TENG prototype through the motion of the oscillatory system can be described at device level as a damped system subjected to a harmonically varying force provided by the mechanical shaker table. Consequently, the $V_{\text {ocmax }}$ at resonant frequency can be expressed as [16, 17] (see Supplementary Information document):

$$
V_{o c \max }=\left(\frac{\sigma}{\varepsilon_{0}}\right)\left(\frac{m_{0} a}{2 k \zeta}\right)
$$

Where $m_{0}$ is the mass of the top plate, $k$ is the stiffness coefficient of each spring, $\zeta$ is the damping coefficient of the DMCS-TENG system and $a$ is the acceleration of the mechanical shaker table. The DMCS-TENG prototypes respond to the mechanical energies applied through resonant oscillatory frequencies. Where the Al foil is in constant nonuniform contact with the dielectric film and attracts electrons away from the copper electrode to flow through the $10 \mathrm{M} \Omega$ 


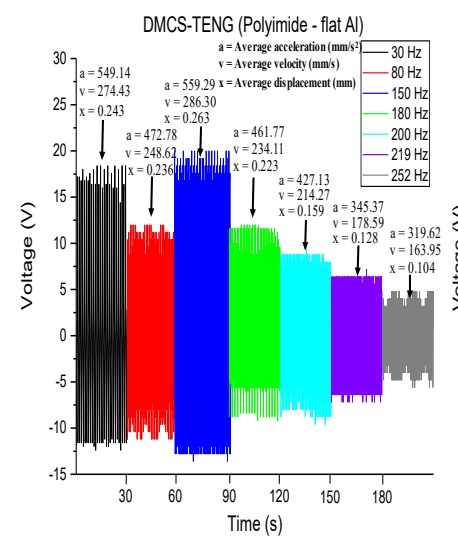

(a)

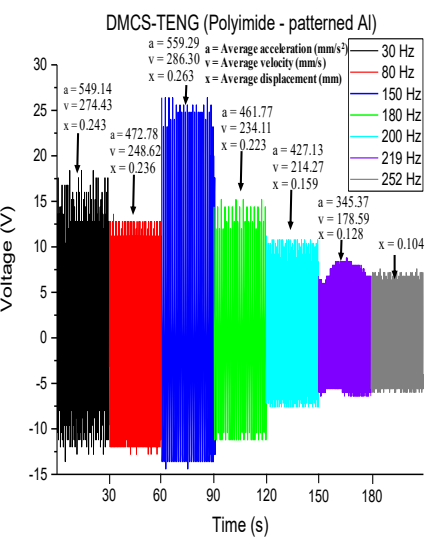

(b)
Fig. 10. DMCS-TENG with (a) polyimide-flat Al and (b) polyimidehoneycomb patterned $\mathrm{Al}$ voltage-time response through simulated resonant frequencies of $30 \mathrm{~Hz}, 80 \mathrm{~Hz}, 150 \mathrm{~Hz}, 180 \mathrm{~Hz}, 200 \mathrm{~Hz}, 219 \mathrm{~Hz}$ and $252 \mathrm{~Hz}$. Average acceleration, velocity and displacement inputs measured with a PDV100 Portable Digital Vibrometer.

external load as shown in Fig 10 (a) and (b) for all the measurements at each frequency over $30 \mathrm{~s}$. It has been observed that the maximum peak-to-peak voltage was measured for the DMCS-TENG prototypes using the honeycomb patterned foil in contact with polyimide (Fig 10 (b)). This can be compared with the output voltage measured using flat $\mathrm{Al}$ foil - polyimide (Fig. 10 (a)).

Among all the DMCS-TENG prototypes employing the top five triboelectric material combinations (Table I (tested)) in contact with flat $\mathrm{Al}$ foil and honeycomb patterned $\mathrm{Al}$ foil, the following results were found. The output power performance comparison between 30 to $252 \mathrm{~Hz}$ with an acceleration between 319.62 to $559.29 \mathrm{~mm} / \mathrm{s}^{2}$ of amplitude showed an improvement factor of 2.3 for the DMCS-TENG devices fabricated using the patterned $\mathrm{Al}$ foil, relative to those fabricated using flat foil (Fig. 11). Such enhancement of the measured values shows the effectiveness of the honeycomb patterned Al foil to improve the electrical contact and surface charge density between the selected triboelectric layers. The maximum generated output voltage $\left(V_{R M S}\right)$ was $12.50 \mathrm{~V}$, with an output current $\left(I_{R M S}\right)$ of $62.27 \mu \mathrm{A}$, which corresponds to the output power and power density of $778.43 \mu \mathrm{W}$ and $12.16 \mu \mathrm{W} / \mathrm{cm}^{2}$, respectively. This was reached using polyimide in contact with the honeycomb patterned $\mathrm{Al}$ foil at the frequency of $150 \mathrm{~Hz}$ and average acceleration of $559.29 \mathrm{~mm} / \mathrm{s}^{2}$. This is followed by the DMCSTENG devices using PDMS and FEP with the output voltage $\left(V_{R M S}\right)$ from $11.10 \mathrm{~V}$ to $4 \mathrm{~V}$, current $\left(I_{R M S}\right)$ from $22.16 \mu \mathrm{A}$ to $63.62 \mu \mathrm{A}$, output power from $246.02 \mu \mathrm{W}$ to $254.48 \mu \mathrm{W}$ and power density from $3.84 \mu \mathrm{W} / \mathrm{cm}^{2}$ to $3.98 \mu \mathrm{W} / \mathrm{cm}^{2}$, in contact with the patterned Al foil (Fig. 11).

The total energy conversion efficiency (ECE) $\eta$ of the DMCSTENG self-resetting system with Polyimide-honeycomb patterned $\mathrm{Al}$ foil was defined as the ratio between the input mechanical energy and the generated electrical energy $E_{\text {ele }}$ that is delivered to the load resistance of $10 \mathrm{M} \Omega$ by the DMCSTENG. The efficiency is determined by (5) [18]:

$$
\eta=\frac{E_{\text {electric }}}{E_{\text {mechanical }}} \times 100 \%=\frac{E_{\text {electric }}}{E_{\text {kinetic }}+E_{\text {elastic }}} \times 100 \%
$$

The $E_{\text {ele }}$ generated by the DMCS-TENG shows an energy output between 0.40 of $13.02 \mu \mathrm{J}$ during a time of $60 \mathrm{~s}$. The

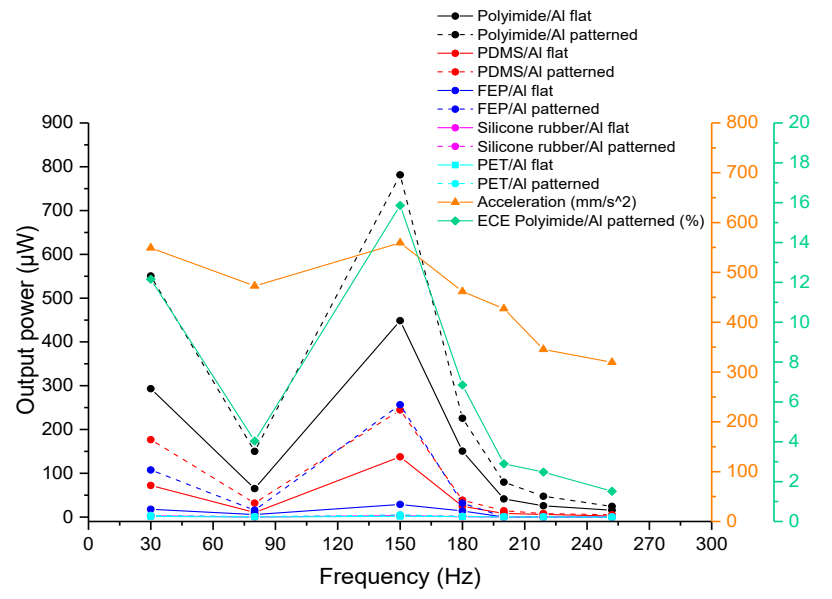

Fig. 11. DMCS-TENG output power, ECE \% and average acceleration measured at the main compressed air bubble frequencies generated during wave impact with polyimide (Kapton), PDMS, FEP, silicone rubber, PET - flat Al foil/honeycomb patterned Al foil.

kinetic $E_{\text {kinetic }}$ and elastic $E_{\text {elastic }}$ energy applied to the DMCSTENG by the mechanical shaker $\left(E_{\text {mechanical }}=E_{\text {kinetic }}+E_{\text {elastic }}\right)$ was calculated by equation (6) and (7). The measured values for the dynamics of the DMCS-TENG were between 319.62 to $559.29 \mathrm{~mm} / \mathrm{s}^{2}$ for the average acceleration $a, 163.95$ to 343.59 $\mathrm{mm} / \mathrm{s}$ for the average velocity $v$ and between 0.10 to $0.26 \mathrm{~mm}$ for the average displacement $x$ of the top dielectric layer. Considering as spring the bending of the top layer, and as constraints the attached kapton tape used to bound four sides of the conductor layer with the dielectric layer that generates the self-resetting system (Depicted in Fig. 9 (a)). The bending stiffness $(k=4$ to $6.14 \mathrm{~N} / \mathrm{m})$ was estimated based in the mass $\left(m_{\text {Polyimide }}=2 g\right.$ ) and average displacement $x$ of the moveable top dielectric layer. $N$ is the number of springs considered for the DMCS-TENG $(N=1)$ (Supplementary Information).

$$
\begin{aligned}
& E_{\text {kinetic }}=\frac{1}{2} m v^{2}=26.87 \text { to } 81.96 \mu \mathrm{J} \\
& E_{\text {elastic }}=\frac{1}{2} k x^{2} N=0.13 \text { to } 0.58 \mu \mathrm{J}
\end{aligned}
$$

The energy applied ( $\left.E_{\text {mechanical }}\right)$ to the DMCS-TENG by the mechanical shaker table was calculated to be between 26.91 to $82.11 \mu \mathrm{J}$, which indicates an efficiency between 1.51 to $15.85 \%$ for the main frequencies of interest between 30 to 252 $\mathrm{Hz}$. The highest efficiency was calculated at $150 \mathrm{~Hz}$ and average acceleration of $559.29 \mathrm{~mm} / \mathrm{s}^{2}$, followed by $30 \mathrm{~Hz}$ with an acceleration of $549.14 \mathrm{~mm} / \mathrm{s}^{2}$ as shown in Fig. 11 according to the experimental measurements.

It was observed, however, that at $80 \mathrm{~Hz}$ with an acceleration of $472.78 \mathrm{~mm} / \mathrm{s}^{2}$ and at frequencies greater than $180 \mathrm{~Hz}$ with acceleration between 319.62 to $461.77 \mathrm{~mm} / \mathrm{s}^{2}$, the output power performance response and efficiency of the devices were lower as shown in Fig. 11. The variation in the output power performance and efficiency (Fig. 11) shows that the proposed devices can effectively respond to a broad range of input oscillating frequencies showing different resonant peaks from 25 to $300 \mathrm{~Hz}$. This means that the device has the potential to produce a higher output power at the moment when the ocean wave impacts the porous structures between $30 \mathrm{~Hz}$ and $50 \mathrm{~Hz}$. After the wave collapses, where the air bubbles are formed 


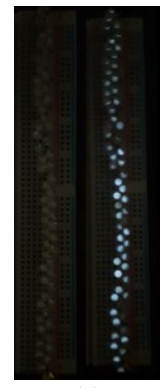

(a)

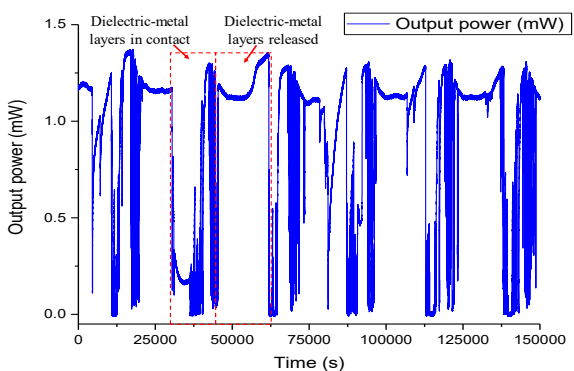

(b)
Fig. 12. (a) Power up of 62 serially-connected white LEDs instantaneously and continuously with the DMCS-TENG prototype (polyimide-honeycomb patterned $\mathrm{Al}$ foil). Measured average instantaneous power consumption by the LEDs was around $13.48 \mathrm{~mW}$. (b) Output power generated by the DMCSTENG prototype (polyimide-honeycomb patterned Al foil), when the dielectric-metal layers are in contact and released exposed to water wave impact with an amplitude of $10 \mathrm{~cm}$ and frequency of $1.2 \mathrm{~Hz}$.

between $150 \mathrm{~Hz}$ and $200 \mathrm{~Hz}$ [2, 3]. The maximum output performance measured experimentally is related to the calculated response obtained by the FEM simulation at $150 \mathrm{~Hz}$ for the proposed energy harvester prototypes, under similar conditions of operation as described in section III.

Additionally, the difference in the output performance between the simulated DMCS-TENG and the real prototypes arises because the FEM simulations were solved by assuming the maximum surface charge density between layers in contact under ideal conditions. This leads to higher output power values (between $3.66 \mathrm{~mW}$ to $416.47 \mu \mathrm{W}$ ). Compared with the measured output power values of the built and tested devices (between $781.31 \mu \mathrm{W}$ to $244.41 \mu \mathrm{W}$ ) in non-ideal conditions.

Moreover, to demonstrate the capability of the DMCSTENG prototypes as direct power source, 62 Kingbright L7104PWC-A $3 \mathrm{~mm}$ white LEDs $(1200 \mathrm{mcd})$ were utilized as operating load connected to the device with the triboelectric material combination of polyimide-honeycomb patterned $\mathrm{Al}$ foil. The peak AC output current of the DMCS-TENG (without rectification) was capable of instantaneously and continuously lighting up 62 serially-connected white LEDs when the device was under $150 \mathrm{~Hz}$ mechanical oscillation and average acceleration of $559.29 \mathrm{~mm} / \mathrm{s}^{2}$ (Fig. 12 (a)). The measured average power consumption by the LEDs was around 13.48 $\mathrm{mW}$ for this demonstration.

Finally, the generated output power by the aforementioned energy harvester, when the dielectric-metal layers are in contact and released under the water wave impact generated in the tank, is shown in Fig. 12 (b). This device with its area of $64 \mathrm{~cm}^{2}$ generated an instantaneous output power, power density of 1.08 $\mathrm{mW}, 169.22 \mathrm{~mW} / \mathrm{m}^{2}$, respectively. Consequently, it is expected to generate output power and energy of $3.05 \mathrm{~W}$ and $2.53 \mathrm{~J}$, if 2,812 of the DMCS-TENG prototypes are tiled (electrically connected) over a water-structure interface with an area of 18 $\mathrm{m}^{2}$ that received wave sizes among $0.3 \mathrm{~m}$ to $4 \mathrm{~m}$ [19].

The total energy (potential energy and kinetic energy) of a wave can be calculated by [20]:

$$
E=\frac{1}{2} \rho g A^{2}
$$

Where $\mathrm{g}$ is the acceleration of gravity $\left(\mathrm{g}=9.8 \mathrm{~m} / \mathrm{s}^{2}\right), \rho$ is the density of water $\left(\rho=1000 \mathrm{~kg} / \mathrm{m}^{3}\right)$ and $\mathrm{A}$ is the wave amplitude $(\mathrm{A}=10 \mathrm{~cm}$, frequency $=1.2 \mathrm{~Hz})$. The input energy $E$, produced by the water wave impact in the tank (Fig. 9 (c)), was found to be approximately $49 \mathrm{~J}$. Accordingly, the DMCS-TENG devices have an efficiency of $5.16 \%$, calculated by:

$$
\eta=\frac{E_{\text {electric }}}{E_{\text {water wave }}} \times 100 \%=5.16 \%
$$

As a result of the power levels achieved by the devices, it is feasible to propose that such energy harvesters can be used to energize wireless nodes for coastal sensing applications.

\section{CONCLUSION}

The proposed work has shown that the triboelectric effect can be used to realize an innovative type of energy harvester for ocean wave impact forces. Firstly, by comparing the findings of the literature, PTFE is the most electronegative material which is widely used in TENG fabrication [12, 21]. Therefore, in our work through an impact test, it was found the ideal dielectricconductor triboelectric material combinations for the fabrication of the DMCS-TENG prototypes. The results showed that silver conductive cloth tape is the material that attains maximum positive charge, and PDMS as the material that attains maximum negative charge. For the same load conditions (10 M $\Omega$ ), it was found that the best output performance was obtained by using dielectric layers with thicknesses between 100 to $125 \mu \mathrm{m}$. Additionally, by employing a mechanical shaker for the DMCSTENG prototypes output power performance characterization. The results show that excellent output power is obtained when using polyimide as the negative material in contact with the honeycomb patterned $\mathrm{Al}$ foil. Such results indicate that the experiments set-up change the contact electrification performance between the dielectric-conductor, which was reflected in the output power performance of the proposed TENG devices. A different most negative material was found compared with the one shown in the literature when the contact is made between dielectric-metal materials $[12,21]$. Such results suggest that the previously published triboelectric series [12, 21] may not give the obvious material pair for the best energy harvesting performance under all circumstances, and for crosscomparison, thickness normalized values must be used.

Secondly, through numerical simulation to obtain a quantitative understanding of the working mechanism and analysis of the output performance of the entire system under the effect of the ocean wave impact oscillating frequencies, the optimum design of the DMCS-TENG was found.

Thirdly, it was observed that the proposed devices' output power and ECE efficiency changes with the main oscillation frequencies between $30 \mathrm{~Hz}$ to $252 \mathrm{~Hz}$ with an amplitude average acceleration between 319.62 to $559.29 \mathrm{~mm} / \mathrm{s}^{2}$. Such variation shows that the proposed devices can effectively respond to a broad range of input oscillating frequencies showing different resonant peaks at the aforementioned frequencies. The maximum output performance was reached using the optimum triboelectric material combination of polyimide - Al foil. Therefore, an enhancement in the output power performance by a factor of 2.3 was achieved using a honeycomb patterned aluminium foil as a low-cost option to enhance the output performance of the DMCS-TENG prototypes. Such improvement in the output performance is due the surface pattern of the $\mathrm{Al}$ foil, which increase the effective contact area 
between the materials that increase the triboelectric charges generated during the friction.

Finally, through the integration of the selected DMCSTENG prototype into the water wave generator tank an output power density of $169.22 \mathrm{~mW} / \mathrm{m}^{2}$ using an area of $64 \mathrm{~cm}^{2}$ was generated. It is predicted that over an area of $18 \mathrm{~m}^{2}$ with wave sizes of $0.3 \mathrm{~m}$ to $4 \mathrm{~m}$, an output power of around $3.05 \mathrm{~W}$ is possible. This offers an alternative approach for generating electrical power for coastal sensing applications that require self-powering. Further investigation is required to explore suitable designs to improve the output electrical performance of the energy harvesting prototypes, with engineering challenges, such as efficient energy coupling and robustness to withstand the harsh environmental conditions of the ocean.

\section{ACKNOWLEDGMENT}

We thank CONACYT for the fellowship awarded to Ulises Tronco Jurado.

\section{REFERENCES}

N. Elvin and A. Erturk, Advances in energy harvesting methods: Springer Science \& Business Media, 2013.

R. B. Mayon, Z. Sabeur, M. Tan, and K. Djidjeli, "Analysis of fluid flow impact oscillatory pressures with air entrapment at structures," Coastal Engineering Proceedings, vol. 1, p. 31, 2017.

R. B. Mayon, "Investigation of wave impacts on porous structures for coastal defences," Doctoral, University of Southampton, 2017.

U. T. Jurado, S. H. Pu, and N. M. White, "A contact-separation mode triboelectric nanogenerator for ocean wave impact energy harvesting," in SENSORS, 2017 IEEE, 2017, pp. 1-3.

Z. L. Wang, L. Lin, J. Chen, S. Niu, and Y. Zi, "Applications in Selfpowered Systems and Processes," in Triboelectric Nanogenerators, ed: Springer, 2016, pp. 351-398.

Z. L. Wang, L. Lin, J. Chen, S. Niu, and Y. Zi, "Harvesting LargeScale Blue Energy," in Triboelectric Nanogenerators, ed: Springer, 2016, pp. 283-306.

U. Khan and S.-W. Kim, "Triboelectric Nanogenerators for Blue Energy Harvesting," ACS nano, vol. 10, pp. 6429-6432, 2016.

Z. L. Wang, L. Lin, J. Chen, S. Niu, and Y. Zi, "Theoretical Modeling of Triboelectric Nanogenerators," in Triboelectric Nanogenerators, ed: Springer, 2016, pp. 155-183.

S. Wang, Y. Xie, S. Niu, L. Lin, C. Liu, Y. S. Zhou, et al., "Maximum Surface Charge Density for Triboelectric Nanogenerators Achieved by Ionized-Air Injection: Methodology and Theoretical Understanding," Advanced Materials, vol. 26, pp. 6720-6728, 2014.

D. Davies, "Charge generation on dielectric surfaces," Journal of Physics D: Applied Physics, vol. 2, p. 1533, 1969.

F. Saurenbach, D. Wollmann, B. Terris, and A. Diaz, "Force microscopy of ion-containing polymer surfaces: morphology and charge structure," Langmuir, vol. 8, pp. 1199-1203, 1992.

J. Chen and Z. L. Wang, "Reviving Vibration Energy Harvesting and Self-Powered Sensing by a Triboelectric Nanogenerator," Joule, 2017.

J. Chen, J. Yang, H. Guo, Z. Li, L. Zheng, Y. Su, et al., "Automatic mode transition enabled robust triboelectric nanogenerators," ACS nano, vol. 9, pp. 12334-12343, 2015.

Y. Yu and X. Wang, "Chemical modification of polymer surfaces for advanced triboelectric nanogenerator development," Extreme Mechanics Letters, vol. 9, pp. 514-530, 2016.

S. H. P. Ulises Tronco Jurado, Neil M. White, "Water-Dielectric Single Electrode Mode Triboelectric Nanogenerators for Ocean Wave Impact Energy Harvesting," in Eurosensors 2018, Graz, Austria, 2018.

R. V. Dukkipati, Vibration analysis: Alpha Science Int'l Ltd., 2004. J. Chen, G. Zhu, W. Yang, Q. Jing, P. Bai, Y. Yang, et al., "Harmonic-resonator-based triboelectric nanogenerator as a sustainable power source and a self-powered active vibration sensor," Advanced materials, vol. 25, pp. 6094-6099, 2013.

[18]

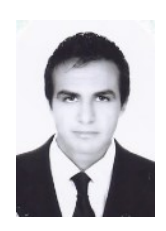

W. Tang, T. Jiang, F. R. Fan, A. F. Yu, C. Zhang, X. Cao, et al., "Liquid-metal electrode for high-performance triboelectric nanogenerator at an instantaneous energy conversion efficiency of 70.6\%," Advanced Functional Materials, vol. 25, pp. 3718-3725, 2015.

Oceanweather.com, ""Current Marine Data | Oceanweather Inc."," http://www.oceanweather.com/data/index.html. , Accessed: 30Oct- 20182018.

A. Khaligh and O. C. Onar, Energy harvesting: solar, wind, and ocean energy conversion systems: CRC press, 2009.

J. Lowell and A. Rose-Innes, "Contact electrification," Advances in Physics, vol. 29, pp. 947-1023, 1980. dots applied to c-silicon solar cells.

$\mathrm{He}$ is currently pursuing the $\mathrm{PhD}$. Degree in Engineering and the Environment with the Mechatronics Research Group, School of Mechanical Engineering, and the Smart Electronic Materials and Systems Research Group, School of Electronics and Computer Science, University of Southampton, Southampton, U.K. His current research interest include triboelectric nanogenerators for ocean wave monitoring applications that required selfpowering.

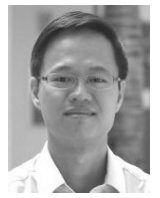

Suan Hui Pu (M'11) received the M.Eng. degree in mechanical engineering from Imperial College London, London, U.K. in 2006 and the Ph.D. degree in electrical and electronic engineering from Imperial College London, London, U.K. in 2010. He is currently an Associate Professor at University of Southampton Malaysia located at Iskandar Puteri, Johor, Malaysia.

$\mathrm{He}$ is a visiting academic in the Schools of Engineering, and Electronics and Computer Science, at the University of Southampton, Southampton, U.K. From 2010 to 2012, he was a Product Engineer with Infineon Technologies (Kulim) Sdn. Bhd. He has published more than 60 peer-reviewed papers in international journals and conferences. His current research interests include NEMS/MEMS sensors and actuators, graphene/graphite sensors and printed electronics.

Dr. Pu has served as a technical committee member for IEEE Electronics Packaging Technology Conference (EPTC) and a reviewer for IEEE Journal of Microelectromechanical Systems, IEEE Electron Device Letters, IEEE Transactions on Components, Packaging and Manufacturing Technology, IOP Nanotechnology, IOP Journal of Micromechanics and Microengineering, amongst others.

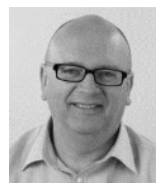

Neil M. White was Head of Electronics and Computer Science from 2011 to 2015 . He obtained a $\mathrm{PhD}$ from the University of Southampton in 1988 for a thesis describing the piezoresistive effect in thick-film resistors. A paper based on this work was awarded the 1989 Educational Prize from the International Society for Hybrid Microelectronics (ISHM). Neil was appointed as a Lecturer within the School in 1990 and promoted to Senior Lecturer in 1999, Reader in 2000 and was awarded a personal Chair in 2002. He is co-author of the book Intelligent Sensor Systems, which was first published by the Institute of Physics Publishing in 1994. He is also co-author of the book MEMS: Mechanical Sensors, published by Artech House.

His research interests include thick-film sensors, intelligent instrumentation, MEMS, self-powered microsensors and sensor networks. He lectures on digital electronics, electronic measurement techniques and advanced instrumentation and sensors. He is a Chartered Engineer, Fellow of the IET, Senior Member of the IEEE, Fellow of the IoP and a Chartered Physicist. He was Chairman of the Instrument Science and Technology (ISAT) group of the IoP from 1997 to 1999. He is a member of the Peer Review College for the EPSRC and is on the Editorial Board of the international journals Sensor Review and Journal of Materials Science: Materials in Electronics. Professor White is also a Series Editor for the Integrated Microsystems series for Artech House.

He has published over 200 scientific papers in the area of sensors and instrumentation systems and holds 10 patents. He is a former Director and cofounder of the University spin-out company Perpetuum Ltd., which specialises in vibration energy harvesting. He was the recipient of the 2009 Callendar sliver Medal, awarded by the Institute of Measurement and Control for his 'outstanding contribution to the art of instruments or measurement'. 